\title{
Besoin d'assistance des médecins de famille et solutions
}

\section{Birgit Laubereau ${ }^{a}$, Pim Krongrava ${ }^{b}$, Manuela Oetterlic, Andreas Balthasard, Stefan Essig ${ }^{e}$, Christian Studer ${ }^{f}$, Carlos Beat Quintog}

${ }^{a}$ Dr méd. et MPH, cheffe de projet, Interface Politikstudien Forschung Beratung

${ }^{b}$ MA, collaboratrice scientifique, Interface Politikstudien Forschung Beratung

${ }^{c}$ dipl. Sc. nat. EPF et MPH, directrice du secteur santé, Interface Politikstudien Forschung Beratung

d prof. Dr, Senior Consultant, Interface Politikstudien Forschung Beratung

e Dr méd. et phil., collaborateur scientifique, Institut für Hausarztmedizin und Community Care Luzern (IHAM\&CC), membre FMH

${ }^{\dagger}$ Dr méd., codirecteur de I'Institut für Hausarztmedizin und Community Care Luzern (IHAM\&CC), membre FMH

${ }^{g} \mathrm{Dr}$ méd., membre du Comité central de la FMH, responsable du département Santé publique et professions de la santé

Jusqu'à présent, les médecins ambulatoires de premier recours reçoivent peu de soutien dans la prise en charge des patient(e)s issu(e)s de la migration. La présente étude a pour objet de cerner les principaux défis et le besoin d'assistance des médecins de famille dans le canton de Lucerne. A la lumière des résultats obtenus, elle formule également des approches de solution pour permettre de relever ces défis.

\section{Contexte}

Le programme national Migration et santé œuvre en faveur de l'organisation appropriée des soins de santé pour les migrant(e)s. Le soutien accordé dans ce cadre a principalement profité au secteur des soins station-

\section{Résumé}

Jusqu'à présent, les médecins ambulatoires de premier recours reçoivent peu de soutien dans la prise en charge des patient(e)s issu(e)s de la migration. La présente étude examine les principaux défis auxquels se trouvent confrontés les médecins de famille en partant de l'exemple du canton de Lucerne et formule des solutions concrètes pour les aider à relever ces défis. Les défis centraux sont les suivants: (1) difficultés linguistiques et financement mal défini des prestations des interprètes, (2) problématique des interfaces avec le secteur social, associée à un manque d'informations chez les médecins, (3) problèmes psychosociaux des patient(e)s et pénuries dans la prise en charge psychothérapeutique et psychiatrique, (4) absence chez les médecins d'informations de fond sur les aspects culturels intervenant dans les soins de santé. Parmi les solutions formulées figurent en particulier la création d'un centre cantonal chargé d'aider les médecins à renvoyer les patient(e)s vers les offres sociales appropriées, un portail Internet présentant des informations sur le thème de la migration et de la santé ainsi que l'intégration de la thématique dans les offres existantes de formation continue, en étroite relation avec la pratique. A l'échelon national, il y a lieu par ailleurs de poursuivre le travail de clarification de la prise en charge des coûts des prestations des interprètes ainsi que d'examiner les possibilités de conseil, de suivi et de prise en charge des migrant(e)s en matière psychosociale. naires depuis 2009 [1]. A contrario, malgré leurs besoins manifestes, les structures ambulatoires n'ont guère bénéficié de mesures de soutien à ce jour [2-4]. Des mesures de soutien ont dès lors été intégrées dans la troisième phase du programme Migration et santé 2014 à 2017 pour aider les médecins de premier recours dans leurs relations avec la population migrante. Dans ce contexte, Interface Politikstudien Forschung Beratung, à Lucerne, et l'Institut für Hausarztmedizin und Community Care Luzern (IHAM\&CC Luzern) ont réalisé une étude pilote conjointe dans le canton de Lucerne. L'objectif de cette étude était, premièrement, de déterminer le besoin d'assistance des médecins de famille dans la prise en charge des patient(e)s issu(e)s de la migration. Le second objectif était, sur la base de ce besoin, d'élaborer des solutions concrètes pour soutenir les médecins de famille.

\section{Méthodologie}

L'étude repose sur quatre éléments méthodologiques. Pour identifier les défis à relever dans la prise en charge ambulatoire des personnes issues de la migration, les auteurs de l'étude ont tout d'abord procédé à une analyse systématique de la littérature spécialisée internationale [5]. Sur cette base, à l'automne 2015, tous les médecins de famille du canton de Lucerne ont été invités, dans un second temps, à participer à un sondage en ligne anonyme. Les thèmes abordés étaient les défis spécifiques, le besoin d'assistance éventuel, l'accepta- 


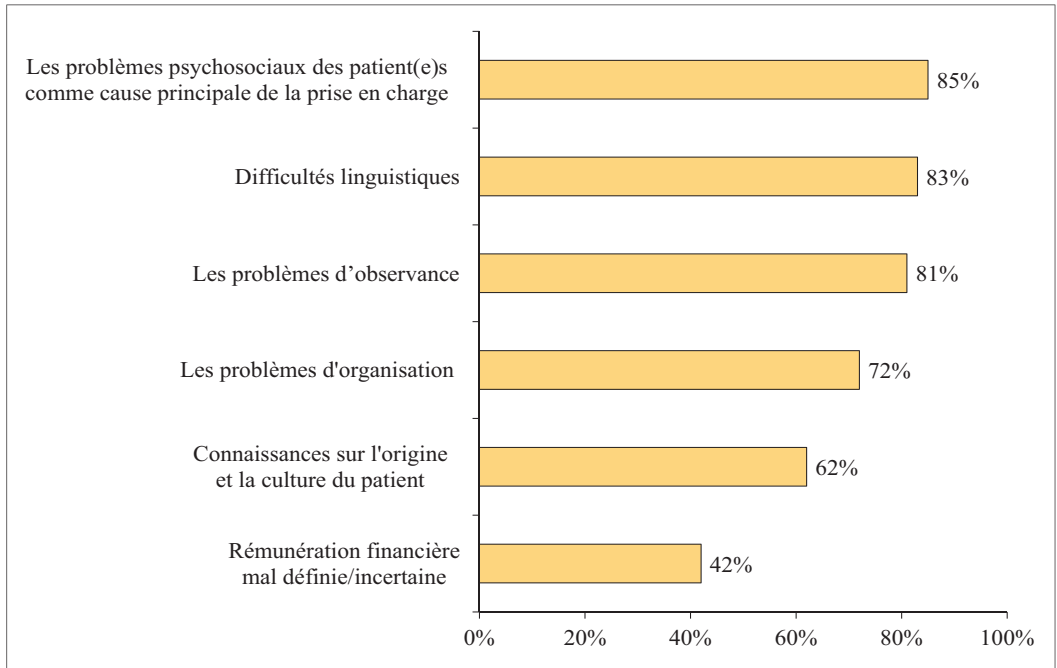

Figure 1: Défis centraux pour les médecins de famille dans la prise en charge des patient(e)s issu(e)s de la migration, sondage en ligne 2015 sur 78 médecins de famille dans le canton de Lucerne.

tion des solutions proposées dans la littérature internationale ainsi que la connaissance et l'utilisation d'un certain nombre d'offres d'aide à l'échelon national et cantonal. Les résultats du sondage ont été approfondis en termes qualitatifs par le biais de cinq interviews exploratoires avec des médecins de famille. Dans un troisième temps, des mesures concrètes ont été élaborées pour le canton de Lucerne dans le cadre d'un atelier rassemblant d'importants acteurs régionaux et cantonaux du secteur social et du secteur de la santé. Enfin, dans un quatrième temps, des recommandations ont été tirées de cet atelier pour le niveau fédéral et pour les autres cantons. Le présent article décrit les principaux résultats de l'étude. Pour une description détaillée de la méthodologie et des résultats, nous vous renvoyons au rapport de l'étude. ${ }^{1}$

\section{Sondage en ligne des médecins de famille lucernois}

Près d'un tiers des médecins de famille contactés (78 sur 267) ont participé au sondage. Il s'agissait de médecins qui traitent au moins une fois par mois des patient(e)s issu(e)s de la migration et dont le profil ne s'écarte pas du profil général des médecins de famille du canton de Lucerne en termes de pourcentage de femmes et de lieu d'implantation du cabinet. Cela étant, les participants étaient légèrement plus expérimentés que la moyenne des médecins de famille du canton. Environ un participant sur deux à l'étude a au moins six mois d'expérience à l'étranger, dans un pays non germanophone dans environ $90 \%$ des cas. Environ $80 \%$ des personnes interrogées travaillaient dans des cabinets employant du personnel plurilingue. Plus http://www.interfaceprojekte/gesundheit// d'un quart des personnes interrogées estimaient que la part de patient(e)s issu(e)s de la migration fréquentant leur cabinet était supérieure à $20 \%$, un pourcentage supérieur donc à la part d'étrangers dans le canton. La patientèle de plus de la moitié des personnes interrogées comprend des patient(e)s en provenance de l'exYougoslavie, d'Afrique, du Portugal et d'Asie.

\section{La communication et les problèmes psychosociaux constituent des défis centraux}

La quasi-totalité des médecins de famille interrogés sont occasionnellement, voire souvent confrontés à des défis particuliers dans la prise en charge des patient(e)s issu(e)s de la migration; seuls environ dix pour cent d'entre eux sont rarement dans ce cas. Les trois défis les plus fréquemment cités: les problèmes psychosociaux des patient(e)s comme cause principale de la prise en charge, les difficultés linguistiques et les problèmes d'observance (cf. fig. 1).

Le besoin d'assistance est important: deux tiers des médecins interrogés ont indiqué qu'ils avaient besoin d'offres d'assistance pour la prise en charge des patient(e)s issu(e)s de la migration.

\section{Les offres existantes sont en partie méconnues}

Les auteurs de la présente étude ont également cherché à savoir dans quelle mesure les médecins de famille lucernois connaissent les offres de soutien existantes, tant nationales que cantonales, et dans quelle mesure ils y ont recours et les trouvent utiles. Le service de consultation sociale de Caritas Lucerne est connu de $90 \%$ des personnes interrogées, utilisé par près de trois quarts d'entre elles et jugé majoritairement utile. Toutes les autres offres de soutien sont nettement moins connues et plus rarement utilisées. Environ la moitié des médecins de famille interrogés ont entendu parler du guide de santé cantonal pour les patients (un peu moins souvent dans le cas du guide national); néanmoins, moins d'une personne interrogée sur quatre y a recours. Seule une personne interrogée sur cinq à peine connaît le service téléphonique national d'interprètes et le site Internet migesexpert.ch de la CRS, qui - il faut le préciser - n'avait été mis en ligne qu'environ trois mois avant le sondage. L'outil d'e-learning «Interaction et qualité», que l'OFSP a mis en ligne à l'été 2014, n'est connu et utilisé que par un très faible nombre de personnes interrogées.

Le sondage en ligne avait aussi pour objet de déterminer dans quelle mesure les médecins de famille colla- 
borent avec certaines institutions du secteur psychosocial et sont satisfaits de cette collaboration. Plus de 80\% des personnes interrogées collaborent avec l'hôpital Luzerner Psychiatrie et Caritas Lucerne et sont satisfaits de cette collaboration dans la grande majorité des cas. Seul près d'un quart des personnes interrogées collaborent avec le service cantonal chargé du conseil et de l'intégration des étrangers et presque toutes sont satisfaites de cette coopération. Ce niveau de collaboration assez faible pourrait être dû au manque de notoriété de ce service auprès des médecins de famille, comme il est apparu dans les interviews.

\section{Solutions pour l'assistance aux médecins de famille}

A la lumière des résultats de l'étude, les quatre défis centraux suivants pour les médecins de famille ont été identifiés: (1) difficultés linguistiques et financement mal défini des prestations des interprètes, (2) problématique des interfaces avec le secteur social, associée, pour les médecins, à une difficulté d'accès aux informations spécifiques fournies par le secteur social, (3) problèmes psychosociaux des patient(e)s et pénuries dans la prise en charge psychothérapeutique et psychiatrique, (4) absence chez les médecins d'informations de fond sur les aspects culturels intervenant dans les soins de santé. Pour chaque défi, l'équipe de projet a élaboré une proposition de solution, l'accent étant mis sur la faisabilité pratique. Ces propositions ont été débattues et concrétisées dans le cadre d'un atelier interdisciplinaire rassemblant une vingtaine d'acteurs importants du secteur social et du secteur de la santé dans le canton de Lucerne (représentants de la médecine ambulatoire de premier recours et d'urgence, de la psychiatrie/psychothérapie, des caisses-maladie, des services sociaux et des consultations sociales, des services de conseil spécifiquement axés sur la migration et de l'administration cantonale). Cet atelier a déjà contribué à améliorer l'interface entre le secteur de la santé et le secteur social. Pour rendre les résultats de l'atelier utilisables au-delà du canton de Lucerne, en novembre 2016, des recommandations générales en ont été tirées, pour la Confédération et les autres cantons, pour éclairer le besoin d'assistance des médecins de famille dans la prise en charge des patient(e)s issu(e)s de la migration. A l'échelon cantonal, l'une des grandes mesures proposées est la création d'un centre devant permettre aux médecins, grâce à sa fonction de triage, de renvoyer leurs patient(e)s vers les offres sociales appropriées pour les questions autres que médicales. En complément, un portail Internet pourrait servir de portail d'entrée à ce centre; ce portail aurait pour objet de regrouper et de fournir toutes les informations sur les offres de soutien dans le domaine médical et social dans le canton ainsi que des supports d'information dans différentes langues. Parallèlement à cela, les offres existantes doivent être utilisées et, le cas échéant, étendues. Par ailleurs, les médecins de famille intéressés doivent se voir proposer des offres de formation continue accessibles sur des thèmes interculturels, idéalement sous la forme d'ateliers proches de la pratique. Il conviendrait à cet égard d'utiliser des formats existants tels que des séminaires nationaux, ainsi que des offres régionales de formation continue comme les cercles de qualité. A l'échelon national, il faudrait en particulier avancer dans la clarification de la prise en charge des coûts des prestations des interprètes dans le secteur ambulatoire, tout en soutenant activement la communication sur les offres de soutien qui existent à l'échelon national. Il conviendrait également de réfléchir au soutien à apporter aux différentes possibilités de conseil, de suivi et de prise en charge des patient(e)s issu(e)s de la migration et présentant des problèmes psychiques et de faire avancer activement cette thématique. Les pénuries dans la prise en charge psychiatrique et psychothérapeutique constituent un problème très actuel, qui touche certes toute la population suisse mais qui s'accentue chez les patient(e)s issu(e)s d'un autre environnement linguistique et culturel. Ce problème devrait encore s'amplifier compte tenu de la dynamique migratoire actuelle, chez les jeunes en particulier.

\section{Remerciements}

Nous tenons à remercier les médecins qui ont pris le temps de répondre au sondage en ligne et qui ont bien voulu se prêter aux interviews. Nous souhaitons par ailleurs adresser nos remerciements aux membres du groupe d'accompagnement ainsi qu'aux participants à l'atelier pour leur éclairage et leur engagement.

\section{Disclosure statement}

Les auteur(e)s ne présentent aucun conflit d'intérêts en lien avec cette étude. L'étude a été financée dans le cadre du programme Migration et santé 2014 à 2017 de l'Office fédéral de la santé publique (OFSP).

\section{Références}

1 http://www.hospitals4equity.ch/index.php/fr/home-fr

2 OFSP, programme national Migration et santé. Bilan 2008-2013 et axes prioritaires 2014-2017. Berne, 2013.

3 Rüefli C. Möglichkeiten zur Unterstützung von Hausärzt(inn)en bei der Betreuung von Patient(inn)en mit Migrationshintergrund. Berne, 2008.

4 Oetterli M, Niederhauser A. Bedarfsanalyse: Migrationsgerechte Angebote im Bereich Gesundheitsförderung und Prävention sowie in der Versorgung des Kantons Luzern. Interface Politikstudien Forschung Beratung. Lucerne, 2012.

5 Christen D. Approaches to improve the interaction between general practitioners and migrants. Master Thesis, Master Program in Health Sciences, University of Lucerne, 2015.

Crédit illustration

(c) Interface et IHAM\&CC, Lucerne. 